\title{
Análise da diversidade genética entre acessos de banco ativo de germoplasma de algodão(1)
}

\author{
Luiz Paulo de Carvalho(2), Marcelo Abreu Lanza( ${ }^{(3)}$, Joel Fallieri(3) e José Wellingthon dos Santos ${ }^{(2)}$
}

\begin{abstract}
Resumo - O conhecimento da diversidade genética entre um grupo de genitores é importante no melhoramento, sobretudo na identificação de combinações híbridas de maior heterozigose e maior efeito heterótico e, portanto, na recuperação de genótipos superiores nas gerações segregantes. Os métodos preditivos de diversidade têm sido muito utilizados porque dispensam a obtenção de combinações híbridas entre os genitores e normalmente usam uma medida de similaridade para avaliar a diversidade entre eles. Foram avaliados 221 acessos do banco ativo de germoplasma de algodão da Epamig, por meio da distância euclidiana média e posterior agrupamento dos indivíduos, de modo a permitir escolhas mais apropriadas de genitores para cruzamentos. Para isso foram consideradas 11 características morfológicas e de fibra. Foi detectada grande diversidade genética entre os 221 acessos reunidos em dez grupos distintos. A maior distância euclidiana (4,36) ocorreu entre os acessos S 8186 e T-295-1-1 e a menor $(0,25)$ entre os acessos TX CACES 1-81 e TX CDPS 177.
\end{abstract}

Termos para indexação: Gossypium, variação genética, genitores, hibridação, método de melhoramento.

Analysis of the genetic diversity among accessions of cotton germplasm collection

Abstract - The knowledge of the genetic diversity among groups of parents is important to plant breeding mainly in the identification of hybrid combinations that show more heterozigosity and higher heterotic effect, and in the recovering of greater genotypes in segregating generations. The predictive methods of diversity have been used considerably because they discard the obtainment of hybrid combinations between parents. They often use a measure of similarity to evaluate the diversity, such as the euclidean distance. In this work 221 accessions of germplasm collection belonging to Epamig were evaluated using the euclidean distance and later grouping the accessions to get an alternative criterion to choose parents for hybridization. Eleven morphological and fiber traits were considered. A great diversity among the 221 accessions was observed and they were clustered in 10 groups. The largest euclidean distance mean obtained (4.36) was between the S 8186 and T-295-1-1 accessions and the smallest (0.25) between the TX CACES 1-81 and TX CDPS 177 accessions.

Index terms: Gossypium, genetic variation, parents, hybridization, breeding methods.

\section{Introdução}

O conhecimento da diversidade genética entre um grupo de genitores é importante no melhoramento, sobretudo para identificar combinações híbridas de maior heterozigose e de maior efeito heterótico. Tomando-se por base essas combinações, a proba-

(1) Aceito para publicação em 25 de julho de 2003

(2) Embrapa-Centro Nacional de Pesquisa de Algodão, Caixa Postal 174, CEP 58107-720 Campina Grande, PB. E-mail: lpaulo@cnpa.embrapa.br, jwsantos@cnpa.embrapa.br

(3) Empresa de Pesquisa Agropecuária de Minas Gerais, Caixa Postal 351, CEP 38001-970 Uberaba, MG. E-mail: mlanza@epamiguberaba.com.br, fallieri@zaz.com.br bilidade de se recuperar genótipos superiores nas gerações segregantes é maior. O uso de genitores com insuficiente diversidade genética na formação de populações para hibridação reduz a variabilidade genética quanto aos caracteres quantitativos (Fehr, 1987). Maurya \& Singh (1977) comentam que os genitores com ampla diversidade genética devem ser usados para produzir melhores tipos segregantes.

Os métodos preditivos de diversidade genética têm sido bastante utilizados, sobretudo pelo fato de que, ao se basearem em diferenças morfológicas e fisiológicas dos genitores, dispensam a obtenção das combinações híbridas entre eles, o que é vantajoso, especialmente quando o número de genitores cujas diversidades se deseja conhecer é elevado. Entre os 
métodos preditivos estão aqueles que quantificam a diversidade por meio de medidas de dissimilaridade, entre as quais encontra-se a distância euclidiana. Considerando-se essas medidas, aplicam-se métodos aglomerativos, estudando-se a diversidade entre os materiais avaliados. A distância generalizada de Mahalanobis e a euclidiana têm sido as mais utilizadas, a primeira necessitando de repetições para ser estimada. A distância euclidiana pode ser estimada tomando-se por base dados sem repetições, como é o caso de dados oriundos do banco ativo de germoplasma, tornando-se viável a sua aplicação. No caso de bancos de germoplasma, o número de acessos é relativamente grande e os dados são coletados, geralmente, no campo, em fileiras simples, sem repetições.

O objetivo deste trabalho foi avaliar a diversidade genética dos acessos de algodão do banco ativo de germoplasma da Epamig, de modo a permitir escolhas mais apropriadas de genitores para cruzamentos.

\section{Material e Métodos}

Em 1998 foi instalado na Fazenda Experimental de Gorutuba, da Empresa de Pesquisa Agropecuária de Minas Gerais, Município de Porteirinha, MG, um experimento composto de 221 acessos do gênero Gossypium. Cada acesso foi semeado em fileira de $10 \mathrm{~m}$, sem repetições, no espaçamento de 1,00 x 0,40 m. Foram avaliadas as seguintes características: porcentagem de fibra, índice de fibra (g), comprimento de fibra (2,5\% mm), índice de uniformidade de fibra (\%), resistência de fibra (índice Pressley), finura de fibra (índice micronaire), altura de planta (cm), precocidade de maturação (dias), altura do primeiro ramo frutífero $(\mathrm{cm})$, número de ramos frutíferos e número de ramos reprodutivos. Os caracteres de fibra foram determinados em uma amostra de 20 capulhos coletados de cada acesso, na colheita; os demais caracteres, como altura de planta, altura do primeiro ramo frutífero, número de ramos vegetativos e número de ramos reprodutivos, foram avaliados utilizando-se a média de 10 plantas ao acaso.

Inicialmente, com os dados dos 221 genótipos e as 11 variáveis, foram calculadas as distâncias euclidianas médias entre cada par de genótipo, do seguinte modo:

$\mathrm{X}_{\mathrm{ij}}=\mathrm{X}_{\mathrm{ij}} / \mathrm{S}\left(\mathrm{X}_{\mathrm{j}}\right)$

em que $S\left(X_{j}\right)$ é o desvio-padrão dos dados do j-ésimo caráter; então

$$
\mathrm{d}_{\mathrm{ii}^{\prime}}=\sqrt{1 / \mathrm{n} \sum_{\mathrm{j}}\left(\mathrm{x}_{\mathrm{ij}}-\mathrm{x}_{\mathrm{i}^{\prime} \mathrm{j}}\right)^{2}}
$$

em que dii' é a distância euclidiana média baseada em dados padronizados e n é o número de caracteres analisados. Obteve-se, assim, uma matriz de distância pxp, em que $\mathrm{p}=221$; em seguida aplicou-se o método aglomerativo do vizinho mais próximo na tentativa de se estabelecer um dendrograma que pudesse estabelecer grupos de genótipos. Utilizou-se o Proc Cluster do SAS, versão 8.2 (SAS Institute, 2000). Posteriormente, selecionaram-se de três a quatro genótipos de cada um dos grupos formados e, ainda, os dois mais e menos divergentes na análise inicial para um novo cálculo de distância euclidiana média e posterior agrupamento, perfazendo o total de 38 acessos, numa segunda análise, mantendo-se, nestes, a variabilidade presente entre os acessos iniciais. De posse da nova matriz de distância euclidiana média, agruparam-se os 38 acessos pelo método do vizinho mais distante.

\section{Resultados e Discussão}

O resumo dos resultados do agrupamento inicial dos 221 acessos feito pelo método do vizinho mais próximo está apresentado na Tabela 1 . O dendrograma resultante desta análise não pode ser apresentado em virtude do elevado número de acessos, contudo, é apresentado na Figura 1 um dendrograma menor com a análise de 38 acessos em que tentou-se manter a variabilidade presente nos 221 acessos iniciais. Os acessos são principalmente de Gossypium hirsutum L., de G. barbadense L., em menor número, e de derivados de materiais silvestres, como os que foram sintetizados para resistência ao bicudo, isto é T-277-2-6, T-295 e outros, de acordo com Lukefahr \& Vieira (1986). A análise realizada considerou todos os acessos a fim de se conhecer a extensão da diversidade genética entre eles. Pela Tabela 1, constatase grande diversidade entre os acessos, revelada pelos dez grupos apresentados. Os materiais do grupo 10 são os mais divergentes pois foram aqueles que apresentaram as maiores distâncias em relação à maioria dos genótipos, como pode ser observado na Tabela 2. Os materiais do grupo 10, T-295, duas linhas dele derivadas (T-295-1-1 e T-295-5-2) e o T-277-13-2, foram selecionados para resistência ao bicudo do algodoeiro e derivam de acessos primiti- 
Tabela 1. Grupos formados pelo primeiro agrupamento dos 221 genótipos de Gossypium spp.

\begin{tabular}{|c|c|c|c|}
\hline \multicolumn{4}{|c|}{ Grupo 1} \\
\hline TX CACES 1-81 & TX CDPS 177 & $137 \mathrm{~F}$ & 3996 \\
\hline TX-LEBOS 1-81 & MG-82570 & C-25-6-79 & Paymaster 303 \\
\hline 73 & Thorpe & (G4009 x G45570)F4b & $4 \mathrm{~S} 180$ \\
\hline SS 186 & La DASS 5194 & S 6046 & T-7538 \\
\hline Tamcot SP 21 & T-8688 & V-79-099 & 6111 \\
\hline DPL 16 Ne AD3 & C-65-7-80 & V-79-090 & V-79-093 \\
\hline C-97-2-81 & $153-7$ & - & - \\
\hline \multicolumn{4}{|c|}{ Grupo 2} \\
\hline TX CAMAS 1-81 & V 79-098 & TX CARBORS 482 & TH 458 \\
\hline Tashkent 2 & SL-26-691 & Stoneville 7A Ne & Stoneville 213 \\
\hline SL-7-Sm6 & Sl-15-60223 & SL-156160 & Sl-15-43273 \\
\hline HD-3 & 3279 & 6396 & Acala Greeg $25 \mathrm{~V}$ \\
\hline G 002-7-11 & Auburn FG-277 & BJ 3127 & C-1211 \\
\hline HG-17-1-81 & SS-153 & Lasani II & - \\
\hline \multicolumn{4}{|c|}{ Grupo 3} \\
\hline BJ 3136 & BJ 3137 & BJ 3146 & 4459 \\
\hline OSF & SL-1(15-43273) & M-73130 (SM3) & SL-23-61142 \\
\hline SL-15-60225 & SL-9-72158 & SL-15-82215 & SL-26-691 \\
\hline SL-2 & SL-22-2223 & SL-22-61131 & SL-9-6142 \\
\hline SL-8-2 & SATU 65 & SL-23-72187 & SL-3-1554 \\
\hline SU-01 & - & - & - \\
\hline \multicolumn{4}{|c|}{ Grupo 4} \\
\hline Auburn 566 RNR & Auburn FG-16 & Coker 310 & Empire WR \\
\hline Coker 3131 & Coker Car Queen & Empire Glandless & BJ 3144 \\
\hline Coker 220 & BJ $1304 \mathrm{~V}$ 18B-15 & C-23-8-78 & BJ 3141 \\
\hline DPL Sm Nectl. & SL-8-1 & SL-26-64142 & SL-7-Sm6 \\
\hline SL-9-6135 & SL-21-62196 & SL-9-6132 & SL-826-67157 \\
\hline SS-200 & V 78-151 & - & - \\
\hline \multicolumn{4}{|c|}{ Grupo 5} \\
\hline 4521 & 74-HG 404 & $149 \mathrm{~F}$ & GLN 1 \\
\hline Des 24-8-Okra & HD-2 & BJ 3191 & Des $56 \mathrm{Ne}$ \\
\hline Pee Dee 8619 & La 453 RKR & Pee Dee 695 & SK 32 \\
\hline SL-23-7013 & SL-23-6879 & SL-7-6662 & Tashkent 1 \\
\hline Pee Dee 875 & SL-23-66133(Sm2) & Stoneville 7A & Reba B-50 \\
\hline SL-23-70132 & SL-23-73602 & SL-23704 & SL-26-6428 \\
\hline SL-23-72001 & SL-3 & SL-21-82691 & SL-23-7220 \\
\hline SL-5(3-5600) & Pee Dee 0113 & SL-26-64209 & - \\
\hline \multicolumn{4}{|c|}{ Grupo 6} \\
\hline (H 4016x5690) F4b & IPEACO & C-28-8-80 & C-97-2-80 \\
\hline Delcot 311 & C-94-2-80 & C-23-3-78 & DPL 16Ne AD3 \\
\hline La DASS 5187 & La DASS 5175 & La DISIS 1213 & JPM 782-459-3 \\
\hline Paymaster 1764 & C-55-2-80 & Sinwalt 82 & SL-23-6680 \\
\hline SL-23-66133(Sm4) & V-79-053 & Stoneville $731-\mathrm{N}$ & $\mathrm{T}-7044$ \\
\hline Tamcot 788 & V-79-086 & V-79-097 & V-79-059 \\
\hline TX-CABUCS 1-81 & FX-CARBORS-482-Fg & SL-23-61143 & V-79-087 \\
\hline V-79-065 & TX CAHUS 31-82 & - & - \\
\hline \multicolumn{4}{|c|}{ Grupo 7} \\
\hline V 79-094 & V 79-120 & V 79-109 & V 79-100 \\
\hline V 79-092 & V 79-088 & V 79-091 & V 79-089 \\
\hline 2421 & V 79058 & SL-25-62275 & SL-23-7022 \\
\hline Super Okra Compacta & Stoneville 256 & Tamcot SP 37 & Tamcot CAMDE \\
\hline N 9311 & La 434 RKR & S 5303 PD 00113 & Des 24-8 \\
\hline HG-DDS-N-11 & JPM 781-78-3 & Lankburn & - \\
\hline \multicolumn{4}{|c|}{ Grupo 8} \\
\hline $108 \mathrm{~F}$ & $138 \mathrm{~F}$ & 1656-71-5C-2 & BJ 3123 \\
\hline BJ 3134 & BJ 3138 & Acala-4-42-A & SLH-6475 \\
\hline Coker 417 & BP 52NC 63 & HG-NC-SM-10-7 & S 8186 \\
\hline SL-18-61114 & SL-24-82885 & V 79-117 & SL-26-6744 \\
\hline SL-2664304 & SU 0450-8909 & SS-207 & Stoneville 7 \\
\hline Upright short fruit branch & BJ 3139 & T 705 & - \\
\hline \multicolumn{4}{|c|}{ Grupo 9} \\
\hline Albar 627 & Allen 333/57 & AH (67) M & BJ 3129 \\
\hline BJ 592 & CA (68) 36 & Carnak & Dendera \\
\hline Giza 75 & Giza 80 & SL-26-70124 & Т 25 \\
\hline T-277-2-6 & $\mathrm{T}-277-5-3$ & T-295-11-4 & T-295-14-1 \\
\hline T-295-6-2 & V 79-096 & - & - \\
\hline \multicolumn{4}{|c|}{ Grupo 10} \\
\hline T-295-1-1 & $\mathrm{T}-295-5-2$ & $\mathrm{~T}-295$ & $\mathrm{~T}-277-13-2$ \\
\hline $\begin{array}{l}\text { Distância euclidiana médi } \\
\text { Distância euclidiana médi }\end{array}$ & $\begin{array}{l}\text { cessos S } 8186 \text { e T- } 29 \\
\text { essos TX CACES } 1-\varepsilon\end{array}$ & 177) & \\
\hline
\end{tabular}


vos de algodão, segundo Jenkins et al. (1978), Lambert et al. (1980) e McCarty et al. (1982). Assim também outros materiais derivados de T-277 e do
T-295, constantes do grupo 9 e que apresentam grandes distâncias euclidianas em relação aos demais (Tabela 2). Todos esses acessos, com a sigla T, são

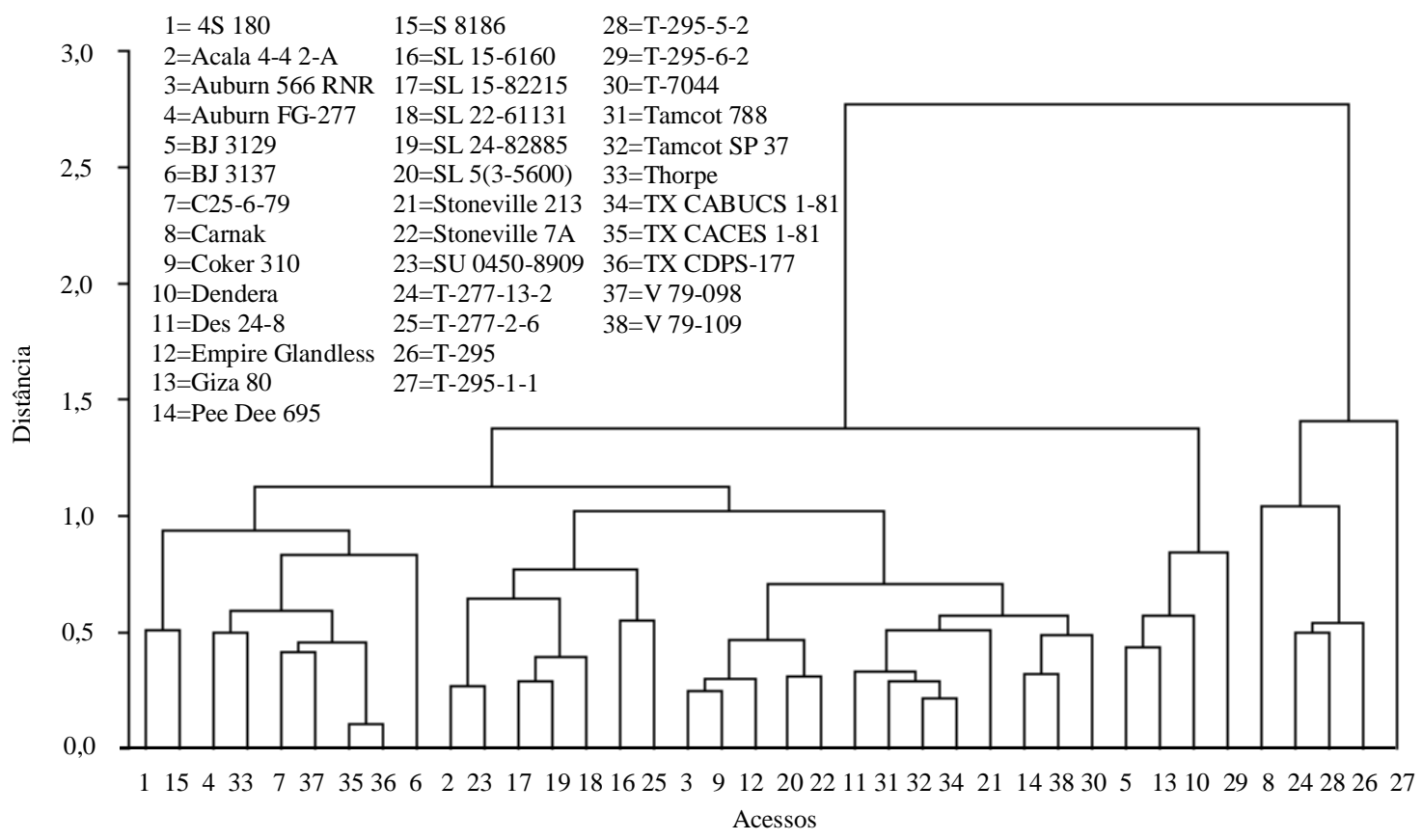

Figura 1. Agrupamento dos acessos de algodão pelo método do vizinho mais distante, com base na diversidade genética expressa pela distância euclidiana média.

Tabela 2. Genótipos mais divergentes entre os 221 acessos baseados na distância euclidiana média, na análise inicial.

\begin{tabular}{|c|c|c|c|}
\hline Genótipos & Distância & Genótipos & Distância \\
\hline T-295-1-1 e demais & $(1,87 \text { a } 4,36)^{(1)}$ & T-277-2-6 e 1656-71-5-C2 & 2,36 \\
\hline T-295-5-2 e demais & $(1,01 \text { a } 3,49)^{(1)}$ & T-295 е C-65-7-80 & 3,09 \\
\hline Carnak e demais & $(1,54 \text { a } 3,56)^{(1)}$ & T-295 e S 8186 & 3,08 \\
\hline T-295 e demais & $(1,01 \text { a } 3,14)^{(1)}$ & T-295 e (G4009 x G4570)F4b & 3,14 \\
\hline T-277-13-2 e demais & $(0,94 \text { a } 3,2)^{(1)}$ & T-277-13-2 e C-97-2-81 & 3,20 \\
\hline 4S 180 e T-277-13-2 & 3,00 & T-277-13-2 е C-65-7-80 & 3,14 \\
\hline AH (67) M e 1656-71-5 C-2 & 2,55 & T-277-5-3 е (G 4009 x G 4570) F4b & 2,49 \\
\hline AH (67) M e C-97-2-81 & 2,51 & T-277-5-3 e V 79-117 & 2,48 \\
\hline Albar 627 e $1656-71-5$ C-2 & 2,35 & T-295-11-4 e (G 4005 x G 4570) F4b & 2,52 \\
\hline Allen 333/57 e 1656-71-5-2 & 2,36 & T-295-14-1 e S 8186 & 2,46 \\
\hline Allen 333/57 e V 79-117 & 2,53 & T-295-14-1 e (G 4009 x 64577) F4b & 2,62 \\
\hline T 295 e BJA 3129 & 2,44 & T-295-14-1 е C-65-7-80 & 2,67 \\
\hline C-97-2-81 e T-227-13-2 & 3,20 & T-295-14-1 e C-97-2-81 & 2,72 \\
\hline C-23-3-78 е Т-277-13-2 & 2,59 & T-295-6-2 e (G 4009 x 64570) F4b & 2,71 \\
\hline 108 F e Giza 80 & 2,37 & T-295-6-2 е C-97-2-81 & 2,71 \\
\hline 108 F e T-277-13-2 & 3,07 & T-295-1-1 e S 8186 & $(4,36)^{(2)}$ \\
\hline 108 F e SL-26-70124 & 2,47 & T-295-5-2-e (G 4009 x G 4570) F4b & $(3,49)^{(2)}$ \\
\hline T-7538 е T-295 & 2,90 & Carnak e C-97-2-81 & $(3,56)^{(2)}$ \\
\hline \multirow[t]{2}{*}{ T-295 e Stoneville 213} & 2,68 & T-295 e (G 4009 x G 4570) F4b & $(3,14)^{(2)}$ \\
\hline & & T-277-13-2 е C-97-2-81 & $(3,20)^{(2)}$ \\
\hline
\end{tabular}

${ }^{(1)}$ Distâncias entre o acesso mencionado e o genótipo mais próximo e o mais distante; a maioria das distâncias mínimas é menor que 2,0. (2)Distância máxima entre cada acesso do grupo 10 . 
originários do Estado do Texas nos EUA, têm em sua genealogia materiais silvestres e ficaram reunidos em grupos mais divergentes (9 e 10) dos grupos iniciais, mostrando serem, como se esperava, os mais divergentes. É evidente que outras análises, considerando-se maior número de variáveis, poderiam subdividir mais os grupos apresentados.

A diversidade genética entre populações pode ser avaliada por meio de estudos genealógicos, diversidade ecogeográfica, análise dialélica com inclusão dos genitores e da análise multivariada, segundo Morais (1992). A falta de registros de ancestrais dificulta o estudo da diversidade por meio de estudos genealógicos. Associações entre diversidade parental e performance da progênie não têm sido bem documentadas em algodão (G. hirsutum L.), conforme Gutierrez et al. (2002). Os autores encontraram correlações entre caracteres agronômicos e de fibra da geração $F_{2}$ com as distâncias genéticas dos genitores, as quais variaram de positivas a negativas, dependendo do caráter, do genótipo utilizado e do ambiente. Quando genitores contrastantes eram usados no cruzamento, havia maior eficiência na predição da performance da geração $F_{2}$ quanto a caracteres agronômicos e de fibra. Esbroeck et al. (1999) não encontraram relação entre parentesco e medidas de similaridade. Singh \& Bains (1968) constataram, em relação ao algodoeiro, que não houve relação entre diversidade genética e geográfica, pois variedades de mesma origem geográfica ficaram em grupos distintos, e atribuíram este fato à seleção e adaptação realizadas nessas populações. Resultados semelhantes em algodoeiro foram obtidos por Amalraj (1982) e Singh \& Gill (1984).

Não é possível correlacionar a diversidade entre os materiais, mostrada pelos dez grupos da Tabela 2, com a sua genealogia, que é desconhecida. Embora cultivares sintetizadas em diferentes localidades ou países apareçam juntas em alguns grupos, cultivares pertencentes a essas mesmas origens se distribuem indistintamente nos grupos, mostrando a importância da seleção e da adaptação dessas cultivares para a sua diversidade. Assim, por exemplo, cultivares de sigla SL desenvolvidas pela Epamig em Sete Lagoas, MG, se distribuem em nove dos dez grupos formados.
Deve-se, contudo, mencionar a importância da genealogia na formação de alguns grupos, como é o caso dos grupos 9 e 10, mais divergentes, que reúnem genótipos originários de materiais silvestres e selecionados para resistência ao bicudo do algodoeiro. McCarty \& Jenkins (2002), ao sintetizarem novas linhas a partir de materiais primitivos cruzados com Deltapine 16, comentam que os acessos derivados são reservatórios de diversidade genética e que devem ser explorados por melhoristas para aumentar a base genética do algodoeiro. Do mesmo modo, as linhas mais divergentes neste trabalho, constantes do grupo 10 e aquelas com a mesma sigla T do grupo 9, foram desenvolvidas pelos mesmos autores a partir também de materiais primitivos cruzados com Deltapine 16. Estas linhas, a exemplo do que sugerem os autores no primeiro caso, têm mostrado grande diversidade, que pode ser usada nos programas de melhoramento, como mostram os resultados deste trabalho, pela total diversidade de quase todos os materiais da coleção. Carvalho et al. (1996) relatam linhagens com alto potencial produtivo obtidas do cruzamento desses materiais de origem silvestre com outros adaptados.

Em uma primeira análise, o intercruzamento dos acessos dos diferentes grupos poderia ser promissor nos trabalhos de melhoramento, em virtude da diversidade entre grupos, mas se deve levar em consideração o potencial per se de cada acesso, sobretudo do rendimento, que não foi avaliado neste trabalho, e outras características desejáveis no melhoramento do algodoeiro; além disso, a origem de cada acesso deve, em termos específicos, ser considerada.

Levando-se em conta as distâncias euclidianas médias indicadas na Tabela 2, vê-se que os genitores mais divergentes foram T-295-1-1, T-292-5-2 e Carnak, os quais apresentaram as mais elevadas distâncias em relação a quase todos os acessos. A maioria dessas situou-se entre 2,0 e 4,36. No agrupamento apresentado na Tabela l, esses materiais permaneceram nos grupos mais divergentes, ou seja, 9 e 10. Considerando-se a análise feita com 38 genótipos, os resultados são semelhantes, conforme a Figura 1, na qual se verifica que o grupo mais divergente permaneceu como o grupo 10 da análise inicial da Tabela 1 , 
ou seja, com os mesmos acessos. O dendrograma com os 221 acessos é muito semelhante ao da Figura 1, em que também se pode dividir os acessos em 10 grupos. Outros pares de acessos mostraramse também bastante divergentes, como apresentado na Tabela 2, e em muitos deles um elemento do par é material de origem silvestre selecionado para resistência ao bicudo. Dependendo de outros caracteres importantes para o melhoramento do algodoeiro, como o rendimento, e caso os genótipos sejam da mesma espécie, os cruzamentos podem ser promissores, com possibilidade de recuperação de tipos superiores nas gerações segregantes.

O melhoramento genético do algodoeiro visa ao aumento do rendimento de fibras, maturação precoce, adaptação à colheita mecanizada, resistência a pragas e doenças e boas qualidades tecnológicas da fibra (Poehlman, 1959). O objetivo final é a obtenção de alto rendimento de fibra e boa qualidade tecnológica. As características apresentadas na Tabela 3 são importantes no melhoramento do algodoeiro; os dados permitem verificar a extensão da variabilidade nestes caracteres pois são apresentados os acessos com maior e menor valor em cada um deles, os quais podem ser utilizados como genitores contrastantes, para estudos de análise genética desses caracteres. A porcentagem de fibra variou de 26,4\% no genótipo Super Okra Compacto, a 41,7\% no genótipo BJ 3127. Quanto ao número de ramos frutíferos por planta, o genótipo T-295 foi o que apresentou o maior valor $(18,0)$. Os acessos com a maior resistência de fibra foram V-79-117 e 4S 180, com 28,0 de índice Pressley, e os genótipos com menores valores foram GH-17-1-81, com 17,8, Coker 3131 e Tamcot SP 21, com valores de 18,1. Quanto à precocidade de maturação em número de dias, o menor valor foi de 93 dias para acessos como Tashkent 2, S 8186, SS 153 e 4S 180. O genótipo mais tardio quanto à maturação foi o T-295, com 111 dias, considerando a média de seis linhagens dele derivadas. A altura de inserção do primeiro ramo frutífero variou de $18,6 \mathrm{~cm}$ do genótipo 1666-71-5-C2 a 49,8 cm do CA (68) 36. Vários genótipos apresentaram índice de fibras curtas com valores menores que 3,8\%; o menor valor foi do acesso T-295-5-2 (2,7\%). A altura de plantas variou de 2,25 m, máxima para o acesso T-295, até 0,77 m, mínima para o 1656-71-5C-2, e nos outros genótipos de 1,23 a 1,25 m.

Tabela 3. Acessos de Gossypium hirsutum spp. analisados, que apresentam maiores e menores médias quanto à porcentagem de fibra (PF), índice de fibras curtas (\%, IFC), índice Pressley (IP), número de ramos vegetativos por planta (NMRVP), altura de plantas (cm, AP), precocidade de maturação (dias, PM), inserção do primeiro ramo frutífero (cm, IPRF) e número médio de ramos frutíferos por planta (NMRF).

\begin{tabular}{|c|c|c|c|c|c|c|c|}
\hline Acesso & $\mathrm{PF}$ & Acesso & IFC & Acesso & IP & Acesso & NMRVP \\
\hline$(\mathrm{BJ}) 3127)^{(2)}$ & 41,7 & SL 2-4-82 885 & 3,8 & $(\mathrm{~V}-79-117)^{(2)}$ & 28,0 & $(\mathrm{BJ} 3129)^{(1)}$ & 1,0 \\
\hline TX Caces 1-81 & 40,3 & $108 \mathrm{~F}$ & 3,8 & 4S 180 & 28,0 & BJ 3127 & 1,2 \\
\hline SL 26-691 & 40,0 & T-295-6-2 & 3,7 & Giza 75 & 27,3 & BJ 3229 & 1,2 \\
\hline $153-7$ & 39,8 & SL-18-61114 & 3,7 & Carnak & 26,5 & C 121-1 & 1,0 \\
\hline La DASS 5194 & 39,6 & 2421 & 3,5 & $153-7$ & 26,0 & Coker 310 & 1,2 \\
\hline TX CDPS 177 & 39,8 & T-295 & 3,4 & T-277-2-6 & 26,0 & SL-34 & 1,0 \\
\hline $153-7$ & 39,8 & T-295-1-1 & 3,0 & Lasani II & 25,9 & SS-186 & 1,2 \\
\hline TX CARBOSR 482 Fg & 39,2 & $(T-295-5-2)^{(1)}$ & 2,7 & Dendera & 26,2 & SL-26-64-209 & 1,4 \\
\hline BJ-3136 & 40,0 & U.S. Fruit. Branch & 3,8 & $(\mathrm{GH}-17-1-81)^{(1)}$ & 17,8 & T-86-88 e outros & 1,4 \\
\hline (Super Okra Compacta) $^{(1)}$ & 26,4 & (Auburn FG-277) $^{(2)}$ & 7,1 & (Tamcot SP 21) & 18,1 & $(\mathrm{~T}-295-1-1)^{(2)}$ & 7,0 \\
\hline Acesso & AP & Acesso & PM & Acesso & IPRF & Acesso & NMRFP \\
\hline 3996 & 1,25 & Auburn FG-277 & 106 & SL-15-60223 & 45,0 & $(\mathrm{~T}-295)^{(2)}$ & 18,0 \\
\hline $108 \mathrm{~F}$ & 1,25 & BJ 3129 & 105 & {$[\mathrm{CA}(68) 36]^{(2)}$} & 49,8 & $\mathrm{~T}-295-1-1$ & 18,0 \\
\hline Des 24-8 & 1,25 & BP 52 NNC 63 & 105 & Carnak & 46,8 & V-79-095 & 17,0 \\
\hline Paymaster 1764 & 1,25 & CA (68) 36 & 105 & Coker 417 & 42,4 & T-295-5-2 & 17,0 \\
\hline SL-22-2223 & 1,25 & Dendera & 106 & SL-26-701224 & 44,2 & T-295-6-2 & 16,4 \\
\hline $4 \mathrm{~S} 80$ & 1,24 & Giza-75 & 110 & SL 3-1554 & 43,8 & $\mathrm{~T}-277-5-3$ & 15,2 \\
\hline Des 56-Ne & 1,24 & Giza-80 & 106 & SL 9-72158 & 42,0 & BJA 592 & 15,2 \\
\hline TH 458 e outros & 1,23 & $\mathrm{~T}-2775-3$ & 106 & T-277-13-2 & 44,0 & Allen 333/57 & 15,2 \\
\hline$(\mathrm{T}-295)^{(2)}$ & 2,25 & $(\mathrm{~T}-295)^{(2)}$ & 111 & T-295-1-1 & 43,8 & $138-\mathrm{F}$ & 15,0 \\
\hline$(1656-71-5 C-2)^{(1)}$ & 0,77 & (Tashkent 2 e outros) $^{(1)}$ & 93 & $(1666-71-5-C 2)^{(1)}$ & 18,6 & $(\mathrm{~V}-79-091)^{(1)}$ & 8,4 \\
\hline
\end{tabular}

(1)Valor mínimo da característica. (2)Valor máximo da característica. 


\section{Conclusões}

1. Existe grande diversidade genética entre os acessos avaliados.

2. Os acessos se agrupam em dez grupos distintos, independentemente de sua origem.

3. Os acessos de origem silvestres selecionados para resistência ao bicudo são os mais divergentes.

\section{Referências}

AMALRAJ, S. F. A. Genetic divergence in Gossypium hirsutum L. Genetic Agriculture, Bangalore, v. 36, p. 2330, 1982.

CARVALHO, L. P. de; LUKEFAHR, M. J.; FARIAS, F. J. C.; VIEIRA, R. de M.; MOREIRA, J. de A. N.; COSTA, J. N. da. Seleção de algodoeiro com resistência ao bicudo. Pesquisa Agropecuária Brasileira, Brasília, v. 31, n. 3, p. 195-199, mar. 1996

ESBROECK, G. A. van; BOWMAN, D. T.; MAY, O. L.; CALHOUN, D. S. Genetic similarity indices for ancestral cotton cultivars and their impact on genetic diversity estimates of modern cultivars. Crop Science, Madison, v. 39, p. 2323-2328, 1999.

FEHR, W. R. Principles of cultivar development. New York: Macmillan, 1987. 536 p.

GUTIERREZ, O. A.; BASU, S.; SAHA, S.; JENKINS, J. N.; SHOEMAKER, C. L.; CHEATHM, C. L.; McCARTY, J. C. Genetic distance among selected cotton genotypes and relationship with $\mathrm{F}_{2}$ performance. Crop Science, Madison, v. 42, p. 1841-1847, 2002.

JENKINS, J. N.; PARROT, J. C.; McCARTY, J. C.; EARNHARDT, T. Evaluation of primitive races of Gossypium hirsutum L. for resistance to boll weevil. Washington: United States Department of Agriculture, 1978. 13 p. (Technical Bulletin, 91).
LAMBERT, L.; JENKINS, J. N.; PARROTT, W. L.; McCARTY, J. C. Evaluation of foreign and domestic cotton cultivars and strains for boll weevil resistance. Crop Science, Madison, v. 20, p. 804-806,1980.

LUKEFAHR, M. J.; VIEIRA, R. M. New sources of boll weevil resistance in primitive races stocks of Gossypium hirsutum. In: BELTWIDE COTTON PRODUCTION RESEARCH CONFERENCE, 1986, Las Vegas. Proceedings... Memphis: National Cotton Council, 1986. p. 493-495.

McCARTY, J. C.; JENKINS, J. N. Registration of 16 day length-neutral flowering primitive cotton germplasm lines. Crop Science, Madison, v. 42, p. 1755-1756, 2002.

McCARTY, J. C.; JENKINS, N.; PARROTT, W. L. Partial suppression of boll weevil oviposition by a primitive cotton. Crop Science, Madison, v. 22, p. 490-492, 1982.

MAURYA, D. W.; SINGH, D. P. Genetic divergence in rice. Indian Journal of Genetics and Plant Breeding, Calcutta, v. 37, p. 395-402, 1977.

MORAIS, O. P. Avaliação do potencial para fins de melhoramento genético, de uma população de arroz oriunda de intercruzamento de variedades, usandose macho-esterilidade. 1992. 255 f. Tese (Doutorado em Genética e Melhoramento) - Universidade Federal de Viçosa, Viçosa, MG, 1992.

POEHLMAN, J. M. Breeding cotton. In: POHELMAN, J. M. Breeding field crops. $2^{\text {nd }}$ ed. Westport: Avi, 1959. $724 \mathrm{p}$.

SAS INSTITUTE (Cary, Estados Unidos). SAS/STAT user's guide. Cary, 2000. CD-ROM.

SINGH, R. R.; BAINS, S. S. Genetic divergence for ginning advance in upland cotton. Indian Journal of Agricultural Science, New Delhi, v. 351, p. 209-213, 1968.

SINGH, T. H.; GILL, S. S. Genetic diversity in upland cotton under different environments. Indian Journal and Plant Breeding, New Delhi, v. 44, p. 506-513, 1984. 